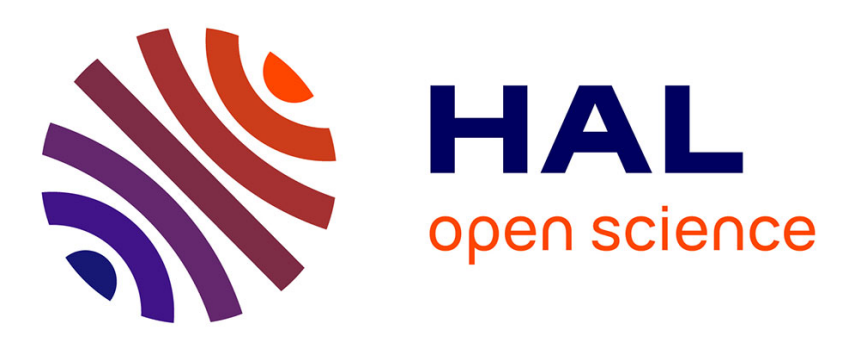

\title{
Diffuse Interface and Strain Anisotropy in Co/Pd(111) Multilayers by Polarized EXAFS Study
}

\author{
S.-K. Kim, Y.-M. Koo, V. Chernov
}

\section{To cite this version:}

S.-K. Kim, Y.-M. Koo, V. Chernov. Diffuse Interface and Strain Anisotropy in Co/Pd(111) Multilayers by Polarized EXAFS Study. Journal de Physique IV Proceedings, 1997, 7 (C2), pp.C2-1097-C2-1099. 10.1051/jp4:19972146 . jpa-00255208

\section{HAL Id: jpa-00255208 https://hal.science/jpa-00255208}

Submitted on 1 Jan 1997

HAL is a multi-disciplinary open access archive for the deposit and dissemination of scientific research documents, whether they are published or not. The documents may come from teaching and research institutions in France or abroad, or from public or private research centers.
L'archive ouverte pluridisciplinaire HAL, est destinée au dépôt et à la diffusion de documents scientifiques de niveau recherche, publiés ou non, émanant des établissements d'enseignement et de recherche français ou étrangers, des laboratoires publics ou privés. 


\title{
Diffuse Interface and Strain Anisotropy in Co/Pd(111) Multilayers by Polarized EXAFS Study
}

\author{
S.-K. Kim ${ }^{(1)}$, Y.-M. Koo and V.A. Chernov* \\ Department of Materials Science and Technology, Pohang University of Science and Technology \\ (POSTECH), Pohang 790-784, South Korea \\ * Siberian Synchrotron Radiation Center (SSRC) of Budker Institute of Nuclear Physics (BINP) \\ Institute of Catalysis, Novosibirsk 630090, Russia
}

\begin{abstract}
We examined interface structure of $\mathrm{Co} / \mathrm{Pd}$ multilayers by quantitative analysis of fluorescence-yield Co $K$-edge EXAFS. To investigate the local atomic structure surrounding Co absorber and its anisotropy in orthogonal directions, two polarization dependent set-ups were used. By means of first shell analysis including distinct nearest neighbors of Co and Pd atoms, it was found that a considerable amount of $\mathrm{Co}-\mathrm{Pd}$ alloy-like phase near interfaces of thin Co layers is formed by atomic scaled diffuseness of growing surface. Moreover, we found Co strain anisotropy depending on Co layer thickness, generally, tensile and compressive strains of in-plane and out-of-plane atoms, respectively. These interface structures might critically affect the tendency of perpendicular magnetization in $\mathrm{Co} / \mathrm{Pd}$ multilayers.
\end{abstract}

\section{INTRODUCTION}

Since an experimental discovery of perpendicular magnetization (PM) in Co/Pd multilayers in 1985 [1], extensive studies have been made to understand the tendency of the PM [2-4]. The magnetic properties of these films might be dominated strongly by the interface structure. The films entirely possess interface widths with anomalous magnetic properties due to repetition of the ultrathin Co layer binding Pd spacers. The presence of interface imperfections is crucial and must be a fundamental factor when explaining strong PM, although clear observation has remained elusive. Thus, we need a detailed interface structure: interfacial mixing and anisotropic Co strains. The polarization-dependent EXAFS technique is an excellent method for anisotropically examining the local structure surrounding specific atoms $[5,6]$. With this detailed structural information, possible mechanisms corresponding to PM should be studied regarding microscopic origins such as the magnetostriction effect of the Co-Pd alloy-like phase. Therefore, we present the interface structure, namely, intermixing and strain anisotropy for $\mathrm{Co} / \mathrm{Pd}$ multilayers, investigated by polarized EXAFS technique.

\section{EXPERMMENT PROCEDURE}

The polarization dependent EXAFS experiments were performed on the EXAFS stage using a Si (111) channel-cut singlecrystal monochromator installed on the VEPP-3 storage ring at the SSRC of the BINP, Novosibirsk [6]. The oscillatory absorption coefficient $\mu(E)$ was measured at the Co $K$-edge $(E=7709 \mathrm{eV})$ in the fluorescence-detection mode with a gas electroluminescent detector filled with Xe gas at near-grazing incidence of synchrotron radiation (SR) for both polarization deponent set-ups: electric vectors $\hat{\varepsilon}$ are parallel (II) and nearly perpendicular $(\perp)$ to the film plane. The $I_{0}$ ion chamber was filled with $5 \% \mathrm{Ar}+$ He gases. To suppress undesirable reflection from $\mathrm{Si}$ wafer, the samples were rotated around the surface normal. $\mathrm{Co}_{0}$ and Pd elements were evaporated from two different heating sources in a vacuum of better than $2 \times 10^{-6} \mathrm{mbar}$ (base pressure is $2 \times 10^{-7}$ mbar). The deposition rate was carefully controlled to less than $0.3 \AA \pm 0.04 \AA$ during whole the preparation process. Low-angle and high-angle $\mathrm{x}$-ray diffraction profiles, and transmission-electron-microscopy crosssection images indicate that the multilayers have well-ordered atomic and layered stacking with (111)-plane preferred crystallographic orientation.

\section{DATA ANALYSIS DETAILS}

The SR is strongly linear-polarized in the plane of the electron orbit. The amplitude of the EXAFS signal depends upon an angle between the $\hat{\varepsilon}$ and the bonding axis from an absorber to a neighbor. This novel characteristic enables us to get anisotropic structural information of radial distances $R$ and coordination numbers $N$ at two polarization dependent set-ups. There are 4 effective nearest neighbors (NN), $N_{\perp}^{*}=4$ in $\hat{\varepsilon} \perp$ the film plane, and 8 effective NN's, $N_{\|}^{*}=8$ in $\hat{\varepsilon} \|$ the film plane for fcc (111) or hcp (0002)-plane oriented polycrystal where total in-plane atoms of $6\left(N_{t o t}^{\text {in }}=6\right)$ and total out-of-plane atoms of $6\left(N_{t o t}^{\text {out }}=6\right), 3$ above and 3 below. For an analysis of two different polarized EXAFS data, two EXAFS functions should be considered and the total EXAFS's contributed by the first shell of $\mathrm{Co}$ and Pd backscatters are given by

${ }^{(1)}$ Present address: Experimental System Group, Advanced Light Source, Lawrence Berkeley Laboratory, 1 Cyclotron Road, CA 94720, U.S.A. 


$$
\begin{aligned}
\chi(k)_{t o t}^{\perp} & =\chi(k)_{C o}^{o u t}+\chi(k)_{P d}^{o u t} \\
\chi(k)_{t o t}^{\|} & =\chi(k)_{C o}^{\text {in }}+\chi(k)_{P d}^{\text {in }} \\
& +\chi(k)_{C o}^{\text {out }}+\chi(k)_{P d}^{\text {out }}
\end{aligned}
$$

For quantitative analysis of the first shell, we fit both $\mathrm{CO}_{0} \mathrm{CO}_{\mathrm{O}}$ and $\mathrm{CO}-\mathrm{Pd}$ peaks in real space simultaneously to the model standards generated by FEFF6 [7] and using UWXAFS3.0 code [8], as shown in Fig. 1. It should be noted that the minor peak of Pd first shell is overlapped with the main peak of the Co first shell. Here we first extracted $\bar{R}_{C o(P d)}^{\text {out }}$ and $N_{C o(P d)}^{o n t}$ as shown in Fig. 1 (a) with Eq. (1), because in-plane atoms do not contribute to EXAFS signals observed in $\hat{\varepsilon} \perp$ the film plane. Bar above symbols means average value over period, i.e., depth $z$. As a subsequent step, four contributions coming from both out-of-plane and inplane backscatters were fit using measured $\bar{N}_{C o(P d)}^{\text {out }}$ and $\bar{R}_{C o(P d)}^{\text {out }}$ with Eq. (2), as shown in Fig. 1(b). Thus, $\bar{N}_{C o(P d)}^{\text {in }}$ and $\bar{R}_{C o(P d)}^{i n}$ can be extracted from radial distribution functions (RDF's) observed in $\hat{\varepsilon} \|$ the film plane. Fourier filtered $k^{3} \chi(k)$ are also shown together with each contribution in Fig. 1 .

Fig. 2 shows Fourier-transforms (FT's) of Co $K$-dge EXAFS data for $[\mathrm{Co}(x \AA) / \operatorname{Pd}(24 \AA)]_{13}$ multilayers, where subscript means the number of bilayers. The same quantitative analysis procedures were employed for those multilayers. The numerical results are shown in Table $I$.

\section{RESULTS AND DISCUSSION}

\subsection{Interface Broadness}

In RDF's at $x=3.2$ and $5.0 \AA$ in Fig. 2 , considerable Pd and $C o$ neighbor peaks appear in both polarizations, which are similar to RDF (see open circles) of Co-Pd alloy film seen in Fig. 1 [9], indicating Co atoms binding Pd spacer layers are scattered into the Pd matrix. Measured values of $\bar{N}_{C o}^{i n}<6$ and $\bar{N}_{C o}^{\text {out }}>0$ in Table I clearly indicate a presence of atomically diffuse heterointerfaces formed during film growth at slightly increasing room temperature. If one monolayer thick $\mathrm{Co}$ atoms were layered on a flat interface, we could observe $\bar{N}_{C o}^{i n}=6$ and $\bar{N}_{C o}^{\text {out }}=0$. By comparing measured $\bar{N}_{C o}^{i n}$ with a calculated one according to the intermixing evolution, the Co concentration $C_{C o}$ profiles within the unit period can be estimated. Assuming a random mixed solid solution, thus $\bar{N}_{C o}^{\text {in }}$ is calculated with respect to the

evolution of intermixing at the interface to be, $\bar{N}_{C o}^{i n}=n^{i n} \int_{\text {period }} C_{C o}^{2} d z / \int_{\text {period }} C_{C o} d z$ with $C_{C o}=\frac{1}{2} C_{\max }[1+\operatorname{erf}(z / \Gamma)]$, where
$n^{i n}=6$ is the total in-plane atoms. The $\Gamma$ is the interface broadness and $C_{\max }$ is the maximum of $C_{C o}$ obeying mass $n^{i n}=6$ is the total in-plane atoms. The $\Gamma$ is the interface broadness and $C_{\max }$ is the maximum of $C_{C o}$ obeying mass (a) $\hat{\varepsilon} \perp$ film plane

(b) $\hat{\varepsilon} \|$ film plane
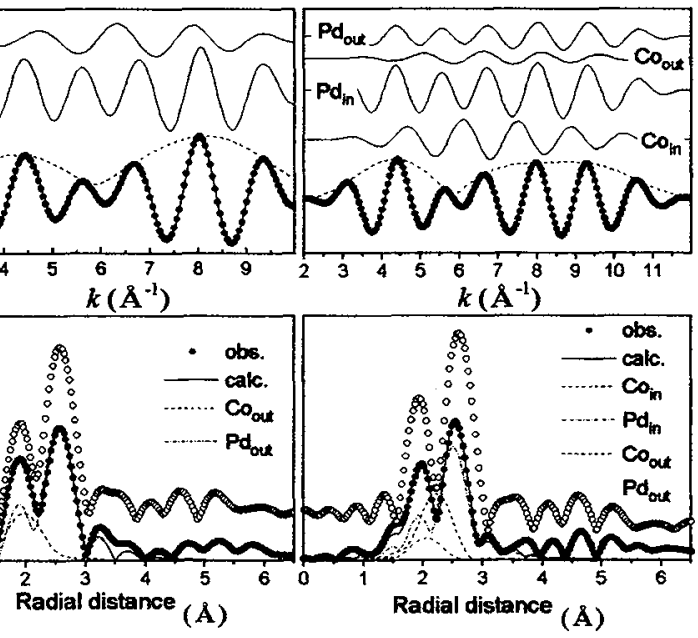

(A)

$\chi(k)$ for the Co $K$-edge EXAFS of [Co(2 A) $\mathbf{P a}(15 \AA)]_{13}$ multilayer, compared to $\mathrm{Co}_{1} \mathrm{Pd}_{3}$ alloy film (open circles), and

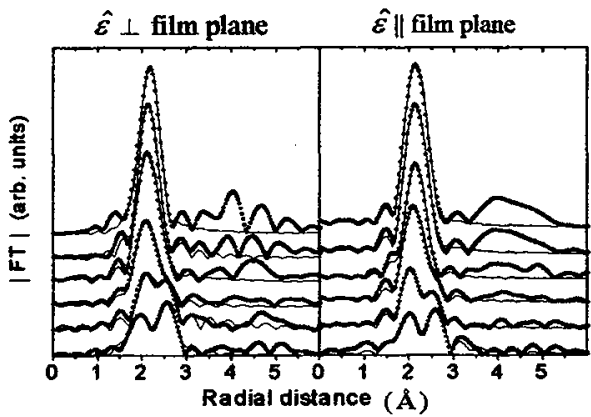

Figure 2 : Fourier transforms of $\mathrm{Co} K$-edge EXAFS for [Co $(x \AA)$ $\operatorname{Pd}(24 \AA)]_{13}$ for both polarization set-ups. $x=3.2,5.0,12.5,16.5$, 23 , and $29 \AA$ are in ascending order from the bottom.

Table I. Best-fit values of Co-Co pairs. Symbol (*) notes that $\bar{N}_{C o}^{\text {in }}$ is fixed at 6 when fit.

\begin{tabular}{ccccc}
\hline$x(\AA)$ & $\bar{N}_{C o}^{i n}$ & $\bar{N}_{C o}^{\text {out }}$ & $\bar{R}_{C o}^{\text {in }}(\AA$ & $\bar{R}_{C o}^{\text {out }}(\AA)$ \\
\hline 3.2 & $1.6 \pm 0.2$ & $2.0 \pm 0.4$ & $2.57 \pm 0.02$ & $2.37 \pm 0.04$ \\
5.0 & $2.8 \pm 0.1$ & $3.5 \pm 0.5$ & $2.60 \pm 0.02$ & $2.36 \pm 0.03$ \\
12.5 & $4.6 \pm 0.3$ & $4.6 \pm 0.2$ & $2.51 \pm 0.01$ & $2.46 \pm 0.01$ \\
16.5 & $5.3 \pm 0.3$ & $5.3 \pm 0.2$ & $2.501 \pm 0.009$ & $2.48 \pm 0.01$ \\
23 & $6^{*}$ & $5.4 \pm 0.2$ & $2.508 \pm 0.006$ & $2.486 \pm 0.007$ \\
29 & $6^{*}$ & $6 \pm 0.2$ & $2.514 \pm 0.006$ & $2.48 \pm 0.02$ \\
\hline
\end{tabular}


conservation law. The plots of calculated $\bar{N}_{C o}^{i n}$ vs. Co layer thickness $t_{c_{o}}$ are seen depending on $\Gamma$ in Fig. 3, compared to the experimental data (solid circles). The inset shows the $C_{C o}$ within a period, determined from estimated $\Gamma$. Fig. 3 shows the quantitative degrees of intermixing and that the $\mathrm{Co} / \mathrm{Pd}$ multilayers deposited at a rate of even less than $0.3 \AA$ have not abrupt interfaces, but rather diffuse ones.

\subsection{Strain Anisotropy}

From the curve-fitting results shown in Table $I$. the change in $\bar{R}_{C o}^{i n(o u t)}$ is plotted as a function of $t_{C o}$ in Fig. 4. Strong strain anisotropy, generally, in-plane tensile and our-of-plane compressive strains are observed at thinner $t_{C o}$. Remarkable relaxation of $\mathrm{Co}_{0}$ atoms toward the bulk value at the thicker $t_{c o}$ might be due to strain relief introduced by misfit dislocations. [6]

\subsection{Correlated Magnetic Anisotropy}

The observed interface broadnesses indicate that Co and Pd atoms are mixed in one or two monolayers width. This demonstrates that the Co atoms do not have broken symmetry by Pd spacers, but have a characteristic Co-Pd alloy-like. This kind of interfacial mixing on atomic scale mainly leads to strong PM together with large strain anisotropy of Co atoms, usually tensile in-plane via magnetostriction effect through its much more negative magnetostriction coefficient than that of bulk $\mathrm{Co}$ by a relation of $K_{\sigma}^{\lambda}=-\frac{3}{2} \lambda_{111} \sigma_{\|}$where $\sigma_{\|}$is the stress applied to $\mathrm{Co}$ atoms in the film plane, and $\lambda_{n}$ is the magnetostriction coefficient of [111] crystallographic direction. The microscopic origin of magnetoelastic anisotropy is similar to that of magnetocrystalline anisotropy coming from spin-orbital coupling. When the stress is applied to the magnetic film, the orientation of magnetization varies according to total energy valence. Detailed description of magnetic anisotropy was made elsewhere [10].

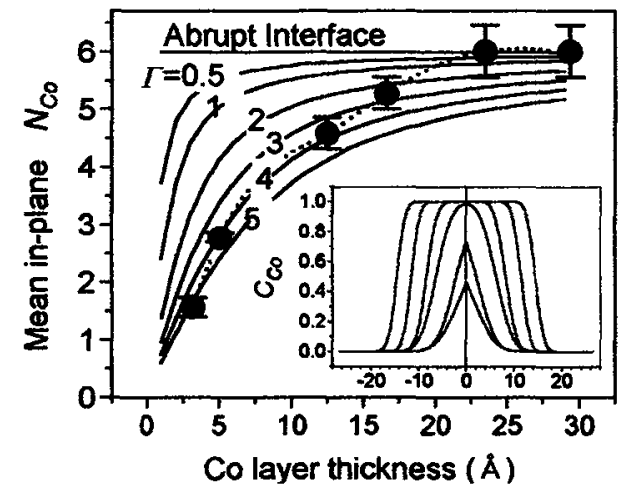

Figure 3: Calculated $\bar{N}_{C o}^{\text {in }}$ (solid lines) depending on $\Gamma$ in $\AA$. Solid circles show experimental data. Inset shows $C_{C_{0}}$ within unit period using determined $\Gamma$.

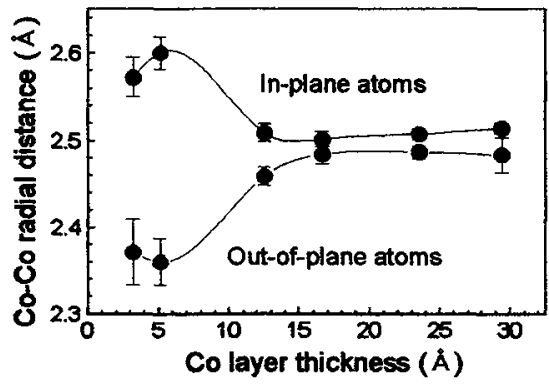

Fignre 4: Ca-Co radial distance versus Co layer thickness

\section{SUMMARY}

Polarization dependent EXAFS is an excellent technique for examining anisotropic local structures such as interfacial mixing and strain anisotropy in multilayers with ultrathin layers. These detailed interface structures in the Co/Pd multilayers should be considered in experiments and theories, which might lead to strong PM via magnetostrictive effect. Finally, the importance of interface structure necessitates further development of depth-resolving EXAFS technique to dinectly differentiate interface structure from the interior of thicker layers.

\section{Acknowledgments}

The authors are grateful to S. G. Nikitenko (Institute of Catalysis, Novosibirsk) for help with EXAFS measurements, and Profs. E. A. Stern and J. J. Rehr for their offers of UWXAFS 3.0 and FEFF 6.0.

\section{References}

[1] P. F. Carcia, A. D. Meinhaldt, and A. Suna, Appl. Phys. Lett. 47 (1985) 178 .

[2] B. N. Engel, C. D. England, R. A. V Leenuwen, M. H. Wiedmann, and C. M. Falco, Phys. Rev. Lett. 67 (1991) 1910.

[3] D. Weller, H. Brandle, and C. Chappert, J. Magn. Magn. Mat. 121 (1993) 461.

[4] T. Takahashi, S. Tsunashima, S. Iwata, S. Uchiyama, Jpn. J. Appl. Phys. 32 (1993) L1328.

[5] P. L. Fèvre, D. Chandesris, H. Magnan, and O. Heckmann, J. De Phys, IV, 4 (1994) C9 159.

[6] S. K. Kim, Y. M. Koo, V. A. Chernov, and H. Padmore, Phys. Rev. B 53 (1996) 11114.

[7] J. J. Rehr, R. C. Alberts, S. I. Zabinsky, Phys. Rev. Lett. 69 (1992) 3397 ; J. J. Rehr, Jpm. J. Appl. Phys. 32 (1993) 8.

[8] E. A. Stern, M. Newville, B. Ravel, Y. Yacoby, and D. Haskel, Phyica B 209 (1995) 117.

[9] S. K. Kim, Y. M. Koo, and V. A. Chernov, Plys. Rev. B (to be published).

[10] S. K. Kim, Y. M. Koo, V. A. Chernov, and D. Weller, Phys. Rev. Lett. (to be published). 